\title{
The Physiology of the Plant Cell*
}

\author{
By Prof. W. Stiles, F.R.S.
}

$\mathrm{I}_{\mathrm{n}}^{\mathrm{N}}$ $\mathrm{N}$ spite of its great importance for the life of man, the physiology of plants is a subject of comparatively recent development. In its earlier phase, during the eighteenth and first half of the nineteenth centuries, it was very largely a study of plant nutrition, from which emerged certain definite information regarding the functions of various plant organs and tissues. It is only more recently that the study of the activities common to all living cells has come to the forefront of physiological inquiry. These activities can conveniently be considered as of two kinds. In the first place all cells respire, in the sense that so long as they are alive, actions proceed in them which involve the release of energy from certain substances. With very few exceptions, these actions take the form of a breakdown of carbohydrate or fat by oxidation to carbon dioxide and water. The second kind of activity exhibited by all cells is to be found in their capacity for absorbing and excreting water and dissolved substances.

It is usually accepted as a fact that every living cell respires, and if this is so, then we must conclude that respiration is something most inextricably connected with life. The general view held regarding the function of respiration, put in as precise terms as possible, is that it provides energy for certain plant movements, and for the building up of substances of higher energy content than the products of photosynthesis which serve as the substrate. While perhaps all plant movements do not obtain the necessary energy for their occurrence from respiratory activity, no doubt some do, and there is every reason for believing that the energy required for the production of various constituents of the plant arises from the same process. But having agreed to this, can we really be satisfied that we have obtained a complete explanation of the function of respiration? In the case of germinating seeds, growing organs, the formation of flowers and fruit, this view seems completely adequate; but we must remember that storage organs, such as potato tubers and carrot roots, respire at a by no means negligible rate and that the same is true of senescent organs such as mature fruit. Indeed, such tissues, notably those of the apple, have provided some of the most interesting data of plant respiration. With

* Based on the presidential address read before Section $K$ (Botany) of the British Association at Cambridge on August 18. what movement, or with what synthesis of materials, is respiration of the cells of the mature apple concerned?

Such considerations lead one to wonder whether respiration is not concerned in some much more subtle way with the maintenance of life. It looks as if the mere maintenance of the protoplasm in a living condition depends on the continuous occurrence of those processes which manifest themselves in the oxidation of organic material to carbon dioxide and water by means of absorbed oxygen. The only exception to this rule is found in certain so-called resting organs, such as seeds, in which the amount of water present is very low, and in which, presumably, the protoplasm is in some very different state from that of active cells.

If we cannot answer this question we can, at any rate, attempt an examination of the functions of respiration of which we feel more certain. The most universal of these is the provision of energy for the building up of materials of higher energy content. The chief problem which awaits solution here is the mechanism by which the energy released in the oxidation of the substrate is transferred to the actions bringing about the synthesis of proteins and other complex plant constituents. A consideration of the relationship between aerobic respiration of apples in air and oxygen and anaerobic respiration in nitrogen led F. F. Blackman to the view that, along with the breakdown of carbohydrate, there is in air a process of oxidative anabolism in which some of the intermediate products of the breakdown of carbohydrate are built back into the system. Evidence for oxidative anabolism in storage tissues such as potato tuber and carrot root has also been obtained in my laboratory by W. Leach, J. K. Choudhury and J. K. Scott, while the investigations of BennetClark on the organic acid metabolism of succulents and other plants has led him to the conclusion that not only does oxidative anabolism occur in these plants, but also that organic acids may quite generally play an important part in this phenomenon.

While then data are accumulating which indicate the linkage of anabolic processes with those of the breakdown of sugar, it is important to note that there is no evidence of the formation of products other than carbohydrates. Is it not possible, however, that syntheses of more complex 
substances are indeed involved, and that we have here a dim glimpse of the mechanism for the production of these substances, and that along with the formation of sugar or some intermediate, there may also be the formation of protein or other complex substances ; that, indeed, we have here the mechanism by which the carbohydrate is brought into a suitable form for combination with nitrogenous and other compounds? However this may be, before we can hope to present a picture of the relation between respiration and vital syntheses, we need not only many more data regarding respiration rates under both aerobic and anaerobic conditions, but also a detailed biochemical analysis of the carbohydrate and various nitrogenous materials present in a wide variety of tissues. So expressed, this may sound a simple enough matter, but actually, as anyone who has attempted to tackle such problems knows, it is one that abounds in difficulties.

While it has generally been assumed that respiration is linked in some unknown way with the synthesis of proteins and other substances, its connexion with those other processes, the absorption and excretion of materials which are characteristic of cells, has only come to be appreciated more recently. The absorption and excretion of water and dissolved substances was generally more or less tacitly assumed to be determined by the physical laws of osmosis and diffusion. Water was supposed to diffuse into or out of the vacuole according to the difference between the osmotic pressure of the cell sap and the sum of the osmotic pressure of the external solution and the inwardly directed pressure of the stretched and elastio cell wall. Dissolved substances were supposed to enter the vacuole according to the laws of diffusion expounded by Graham and Fick more than eighty years ago.

Although Collander's work on the absorption of a number of non-electrolytes indicates that this assumption may, in the case of such substances, be quite justified, it has been known now for thirty years that the entry of electrolytes into cells cannot be explained as the simple diffusion of a substance through a membrane from a region of higher concentration to one of lower concentration. During the years 1909-19, two facts militating against this simple view became established. In the first place, it was shown that the two ions of a salt could be absorbed by plant tissue at different rates, while in the second place it was shown that absorption of a salt or its ions takes place towards a condition of equilibrium which is not that of equality of concentration attained inside and outside the cell, but which depends on the concentration of the salt. With dilute solutions the concentration attained inside the cell may be many times that of the solution outside, while in concentrated solutions the reverse is the case and the concentration of the salt inside, even after forty-eight hours' immersion of the tissue in the solution, may be very much less than that outside. Thus, while more salt is actually absorbed from a stronger solution than from a weaker one, the absorption relative to the concentration is less, both as regards rate and total amount, from a stronger than from a weaker solution.

Various possible mechanisms have been suggested to explain this relation between concentration and absorption. When this problem was first investigated by $\mathrm{F}$. Kidd and myself, we found that the relationship between concentration of salt and absorption was much the same as it would have been if the salt were adsorbed by an adsorbent within the cell. It is easy to suggest that a first stage in the absorption of salts by plant cells is the adsorption of the ions of the salt by some constituent or constituents of the protoplasm. While I have pointed out the similarity of the absorption of salts by plant cells with an adsorption phenomenon, I have more than once stressed the point that this similarity is in itself not sufficient to justify the advocacy of an adsorption theory of salt absorption. Yet it must be admitted that later work by more exact methods has only served to confirm the approximate similarity of the relationship between salt absorption and adsorption. Reference in this connexion may be made to the work of Laine on the absorption of manganese and thallium by roots of Phaseolus multiflorus, as well as to observations of my own on the absorption of sodium chloride by carrot root.

Of course, if the similarity between the relationship of salt absorption to concentration and the adsorption equation is more than a coincidence, adsorption can only be the first stage in this absorption, at any rate by actively growing tissues in which the absorbed ions must be transferred elsewhere. Again, one would expect the adsorbing material to be present in the protoplasm, whereas a number of more recent observations by various investigators indicate that there is actually an increase in concentration of the absorbed ion in the vacuole. The adsorption would then have to be followed by elution of the salt at the surface of the vacuole. In this connexion it is interesting to note that S. C. Brooks has obtained some evidence that Valonia, immersed in sea-water containing rubidium chloride, accumulates rubidium in the protoplasm for two days, after which this cation passes from the protoplasm to both vacuole and external solution. The same worker has also found that when cells of Nitella are placed in 
$0.01 M$ solutions of radioactive potassium chloride, there is an accumulation of potassium in the protoplasm after six hours, before any appreciable amount of potassium appears in the vacuole. Previously, M. M. Brooks had found that when Valonia is immersed in a solution of methylene blue, the cell wall and protoplasm become deeply stained by the dye before any appreciable coloration of the vacuole is observable.

Another mechanism which has been suggested as possibly operative in the absorption of salts is one of interchange of ions within and without the cell under conditions which give rise to the ionic distribution between the cell interior and exterior characteristic of what is called Donnan equilibrium; and in this connexion it must be emphasized that just as adsorption must take place if the cell contains adsorbents of ions capable of reaching the adsorbent, so, if the cell system involves the conditions giving rise to Donnan equilibrium, it is inevitable that the movement of ions demanded by these conditions must result.

The possibility that respiration has a direct effect in bringing about the absorption of ions has been pointed out by several workers, notably by Briggs and S. C. Brooks. The production of carbon dioxide in the cell leads to the appearance of carbonic acid, and hence of its ions, hydrogen and bicarbonate $\left(\mathrm{H}\right.$ and $\left.\mathrm{HCO}_{3}\right)$. The interchange of ions required by the Donnan equilibrium will lead to the diffusion out of hydrogen ions, which are replaced by cations from the external medium, while bicarbonate ions will be exchanged for anions from the external medium. As the tissue continually respires, the production of hydrogen ions continues to replace those which diffuse into the external solution, and so the absorption of ions continues as part of an interchange between tissue and external medium.

Against the view of a direct effect of respiration on salt intake by ionic exchange, it has been urged by Hoagland and Steward that accumulation of ions is negligible or slight when tissue is deprived of oxygen, although there may be a considerable anaerobic production of carbon dioxide. But as regards this objection it must be noted that under conditions of anaerobiosis the rate of carbon dioxide production usually falls rapidly with time, so that it is doubtful whether a considerable production of carbon dioxide anaerobically generally continues for any length of time. The question is obviously one requiring further experimental investigation.

That the absorption of salts by tissues is related to a supply of oxygen, and probably in some way to respiration, there can, however, be no doubt. For more than thirty years it has been known that the growth of plants in water-culture is often accelerated by aerating the solutions, while more direct evidence of the effect of oxygen on the salt relations of cells has been obtained in work with storage tissues. In 1927, I pointed out the importance of maintaining the supply of oxygen to such tissues for the maintenance of their vitality, and that in the absence of an adequate oxygen supply exosmosis of electrolytes took place, leading to the speedy death of the tissues; whereas with maintenance of a supply of oxygen, absorption of electrolytes continued, in the case of beetroot, for example, for periods of about three weeks. Towards the end of this time a condition of equilibrium was reached or approached, in which the content of electrolytes in the external liquid was very low. During this period conditions leading to lower oxygen and higher carbon dioxide concentration led to increase in the electrolyte content of the liquid, while addition of fresh oxygen led to a decrease.

In similar experiments carried out by Briggs and Petrie in 1931, in which a continuous stream of air was passed through the liquid, these workers examined the course of respiration along with the changes in electrolyte content of the external solution, and established the fact that there is a general parallelism between the rate of respiration of the tissue and the electrolyte concentration of the external liquid. If the stream of air was replaced by nitrogen the respiration rate increased, and so did the concentration of electrolytes in the solution, while replacement of the nitrogen by air brought back the original distribution of electrolytes between tissue and external liquid. Steward and collaborators have shown that reduction of the oxygen supply to storage tissue of potato, carrot and artichoke below a certain value limits the accumulation of both the ions of potassium bromide by the tissues, while Hoagland and Broyer have obtained a similar result with barley root systems.

In attempting to explain this effect of oxygen, one must bear in mind that the relationship between respiration and salt accumulation may not be a direct one. The maintenance of an adequate supply of oxygen is necessary to maintain the vitality of the tissue, possibly on account of the deleterious effects of the products of anaerobic respiration. Thus the fact that accumulation depends on oxygen supply may be regarded as an expression of the fact that under conditions of partial or complete anaerobiosis, the functioning of all or many vital processes dependent on the protoplasm is adversely affected, and along with them that of salt accumulation. From this point of view the effect of conditions leading to poor oxygen supply may be related not only to oxygen concentration, but also to accumulation of carbon 
dioxide and other products of anaerobic respiration.

From a consideration of all the data, it seems to me that the following conclusion can be drawn regarding the relationship of respiration to the absorption of salts by plant cells, namely, that accumulation of salt depends on the vitality of the cells and that the maintenance of this vitality depends, as has been long recognized, on the presence of oxygen, either because aerobic respiration or some other process requiring oxygen is essential for this maintenance of vitality, or because in the absence of oxygen the accumulation of carbon dioxide and other products of anaerobic respiration adversely affects the functioning of the protoplasm. This dependence of absorption of salts on the vitality or healthiness of the tissue was clearly shown by my experiments of 1927 , and by the later ones of Steward in which stress was laid on the effect of aeration of the tissues. I think Hoagland's observations on the effect of light on absorption of potassium bromide by Nitella fall into line with these. It was found that absorption of bromide only took place if the cells were exposed to light, or if they had previously been exposed to adequate illumination. If for some time previously they had been growing in weak light, no accumulation of ions took place. Nitella kept for some time in low light is probably somewhat unhealthy, just as is tissue that is deprived of an adequate supply of oxygen. In other words, most of the work published on the relationship between respiration and salt accumulation does no more than show that this accumulation is a vital process, depending on the normal functioning of the protoplasm. Any general relationship between respiration and salt accumulation, as regards the linkage of reactions involved, or the transfer of the energy required for the entry of a salt against its own diffusion gradient, may thus be very indirect.

Thirty years ago, when the importance of the principles of chemical dynamics in life processes was coming to be fully realized, it looked as if the solution of many of the problems of plant physiology, in terms of physical chemistry, was fairly imminent. But with the application of these principles to our investigations into living processes, we find that in every one of them the protoplasm introduces a factor which renders these processes not readily explicable in this way. Clearly we must seek an explanation in the apparent divergence of vital processes from physical or chemical laws in the constitution of the protoplasmic system, and hence a fuller analysis of this system now appears to be a requisite for further advance in our understanding of physiological processes in general. There is at present no reason to suppose that with further advance in knowledge of the protoplasmic system, we shall not ultimately be able to explain physiological processes in physico-chemical terms, and I would re-affirm what F. F. Blackman emphasized in his presidential address to Section $\mathbf{K}$ thirty years ago, namely, "the inevitableness of physicalchemical principles in the cell".

It is scarcely necessary to emphasize how the principles of general cell physiology must be of fundamental importance in plant metabolism, for inasmuch as this depends on the activity of specialized cells and tissues, these, wherever they are alive, must also exhibit the normal features characteristic of protoplasmic activity. But in spheres of botanical science outside the range of pure physiology, the general physiology of the cell is just as important. This applies in particular to ecology. This study, in so far as its aim is the determination of the relationship of plants to their environment, is indeed nothing else than physio$\operatorname{logy}$, a fact which was clearly recognized by Clements more than thirty years ago.

Of the two groups of factors which determine the distribution of vegetation, the climatic and edaphic, the mode of action of the latter in particular can only be studied with any hope of success by those with an adequately deep knowledge and appreciation of cell physiology. It does not need a knowledge of physiology, it is true, to determine plant distribution, but such knowledge is essential for what Tansley, in a paper read to Section K thirtyfour years ago, called "the higher branch of ecology, i.e., the detailed investigation of the functional relations of plant associations to their surroundings". However desirable and necessary the collation of knowledge of plant distribution may be, I am certain that the solution of the fundamental problems of ecology will only be achieved by the use of physiological methods, and particularly by the application of our knowledge of the general physiology of the cell. For edaphic factors must act through the root and by the absorption of materials from the soil, or the exchange of material between the soil and root.

Certain aspects of mycology have much in common with physiology; indeed, that part of mycology which concerns pathogenic organisms is inevitably closely linked with problems of the relation of host and parasite, problems which are, in their very essence, physiological. Years ago it was questioned whether the physiology of the plant physiologists was not half pathology. Certainly the reverse question can be answered with more assurance; pathology is at least partly physiology, and therefore the principles of general cell physiology must here also be of immense importance, and an intimate acquaintance with 
these principles should be an important part of the equipment of the plant pathologist.

Perhaps no branch of botany has made such spectacular advances in recent years as that of cyto-genetics. At least it has produced a nomenclature which rivals or excels the early efforts of the descriptive ecologists ; and just as descriptive ecology can do little more than correlate certain types of vegetation with certain environments, so cytology can do little more than correlate visible structures in the cell with genetical behaviour. I cannot help thinking that a real insight into these problems also will only come with the interpretation of cytological observations in physiological terms, and that the greatest advance in the study of cytology will come with the linking up of the knowledge of the cell acquired by these two lines of investigation, the cytological and physiological. It is surely a rather remarkable fact-one indicating how far away we are at present from the achievement of this end-that the physiologist tends to think of the cytoplasm as the essential factor in determining vital activities, while the cytologist concerns himself almost exclusively with the nucleus. Neither the physiologist nor the cytologist appears at present to have anything but the vaguest ideas of the relationship between the two, a relationship which, however, we may feel sure is most intimate and fundamental to life.

We all know, but it cannot be too strongly emphasized, that botany is the pure science of a great part of the most important industry of the world, agriculture, and that this, like every other industry, can only be carried on wisely if its practice is based on scientific principles. Almost all branches of botany are important for agriculture ; but mycology, genetics, and physiology are particularly so, and certainly physiology is not the least of these. Absorption of water and nutrients from the soil, assimilation of carbon, water relations of the plant, vegetative development, flowering and fruiting, are all problems of agriculture and forestry which are essentially physiological.

But besides these more obvious economic applications, there are numerous industries in which the principles of general cell physiology are no less fundamental. There are all those industries, ever increasing in number and importance, which are based on some particular plant product, such as cotton, linen, jute, rubber, tea, sugar and tobacco, to mention only a few of the more important. Apart from the growing of the plants themselves, which like any other form of agricultural practice should be based on sound physiological principles, a knowledge of these principles may be equally important in the subsequent treatment of the plant material. In particular, a knowledge of cell organization, the action of enzymes contained in the cell, its behaviour towards various reagents, all aspects of general physiology, is essential. Finally, the great food storage industry depends greatly on the application of knowledge of cell physiology. As an example of this I may refer to pioneer work on the scientific principles of cold storage by Jørgensen and myself carried out some twenty years ago. From a consideration of what was then known of the constitution of the cell, we concluded that the satisfactory preservation of certain tissues in the frozen condition depended on rapidly freezing the tissues, a method which was afterwards put into practice in certain branches of the food storage industry. This is, of course, only one instance of the bearing of general cell physiology on the subject of food preservation. The effect of the conditions of storage on enzymes and other cell constituents, and on the vitality of different kinds of cells, tissues and organisms are among the problems which a knowledge of the facts and methods of general cell physiology will help to solve.

With the ever-increasing mass of knowledge in the various branches of botany, an increase which is especially noticeable to-day in those aspects of our subject which are undergoing rapid development, namely, physiology, mycology and genetics with cytology, it is impossible for anyone to be an active worker in more than a relatively very small field of botanical endeavour. We sometimes meet with reference to a mysterious gentleman called the 'general botanist' who is expert in general botany, as someone distinct from the morphologist, physiologist, mycologist or other worker in a defined field. But in these days, when to make any contribution to knowledge necessitates special ization, there can indeed be no such person as the expert in 'general botany', for there is, indeed, no such subject. But in whatever part of our subject our own special interests may lie, we can still appreciate the efforts and aims of workers in other fields, and realize the bearing of work in these fields on our own problems, and in this sense we are all general botanists ; that is, just botanists.

For if 'general botany' as something distinct from 'botany' is a myth, there is no doubt that the various branches of our subject are related in the whole. I have here tried to indicate not only the scope and present position of our knowledge of the general physiology of the cell, but also where this particular part of the science of plants comes into contact with other branches of botany, and how the application of a knowledge of the facts, principles and methods of cell physiology may be expected to lead to an increase in knowledge, not only of the physiology of the plant, but also of other aspects of botanical science and of its industrial applications. 